\title{
Research Application of Temporal Description Logic Algorithm based on Ontology
}

\author{
Xin Zhang ${ }^{1, a}$, ChaoGang Wang ${ }^{1, b}$, Rui Wen ${ }^{2, c, *}$ \\ ${ }^{1}$ Faculty of Humanities, Yunnan, College of Economic and Management, Kunming 650106, China \\ ${ }^{2}$ Department of Computer Science, Yunnan, Yuxi Agriculture of Career Technical College, Yuxi \\ 653106, China \\ a54332254@qq.com, b39266097@qqcom, cruiruiwen@163.com
}

Keywords: Ontology, Temporal description logic algorithm, Application Research.

\begin{abstract}
With the continuous development and extensive application of ontology research, the temporal information needs to be processed, but such ontology description logic has good ability of expression, algorithmically undecidable, simple and shortcomings of inability to describe some concepts in the reality. In this situation, this paper presents temporal specific areas based on time interval, which requires it must be - admissible and add it to the description logic, proposes a new temporal description logic algorithm based on ontology, by proving satisfiability of temporal domain specific is decidable, and verifies the feasibility of the algorithm.
\end{abstract}

\section{Ontology}

Ontology as a concept model which can describe information on semantic and knowledge level modeling tool, since it was put forward, has attracted many researchers attention, and has been widely used in many fields of computer, such as knowledge engineering, digital library, software reuse, information retrieval and heterogeneous information on Web processing, semantic Web. The concept of ontology $=$ Concept + Property + Axiom + Value + Nominal, Ontology is a concept model used to describe a certain domain. From mathematics, Semantic network is a labeled directed graph. Figure three is ontology learning framework. OWL provides three increasingly expressive sublanguages designed for use by specific communities of implementers and users [1-2].

For example, semantic web can express one sentence, such as, my car is red. But Ontology obvious doesn't fit this expression, but for the whole content. Semantic web has no special request for model, while Ontology has five needs: Meta language, class, relation, function, axiom and case, in which axiom can be seen as restraint.

\section{OWL Ontology}

An OWL Ontology consists of a series of axioms and facts, and then adds other Ontology references. Ontology usually has a non-logical note, which contains other non-logical information of the author and relative ontology [3]. OWL ontology is the Web document, and URI can be used for reference. The following is using OWL abstract grammar to explain ontology structure.

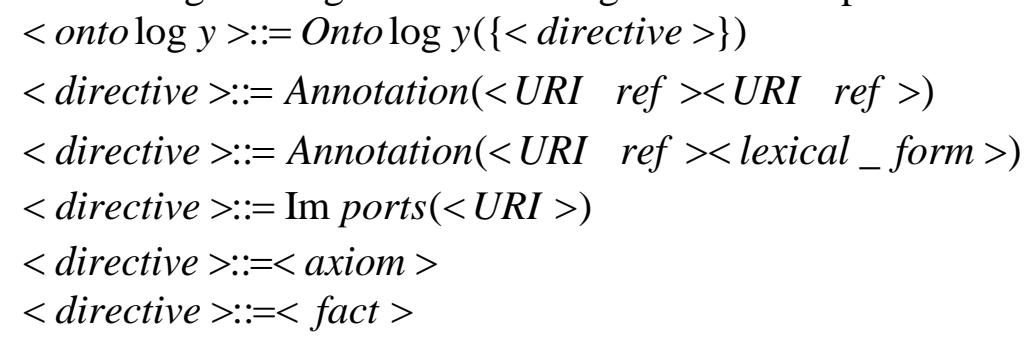

In the abstract grammar of OWL, there are two kinds of facts, one of which is used to indicate a special individual instance information, but an individual instance is given an indi ID, used to mark individual. But not every individual instances is required to be given an indi ID. The individual which is not given an indi ID is anonymous, and cannot be reference for others. 
In the OWL axioms, axiom is mainly used to show equal or sub class, or the public part of the super class sets.

$$
\begin{aligned}
& <\text { axiom }>::=\text { Class }(<\text { classID }><\text { mod ality }>) \\
& \{<\text { annotation }>\}\{<\text { description }>\} \\
& <\text { mod ality }>::=\text { complete } \mid \text { partial } \\
& <\text { axiom }>::=\text { disjo int } \text { with }(<\text { description }>\{<\text { description }>\}) \\
& <\text { axiom }>::=\text { sameClassAs }(<\text { description }>\{<\text { description }>\}) \\
& <\text { axiom }>::=\text { subClassOf }(<\text { description }>\{<\text { description }>\})
\end{aligned}
$$

With the development of information retrieval technology, combined with artificial the intelligent application of technology in the information retrieval, with the intelligent information retrieval technology application, intelligent information retrieval shows off, which is now the information retrieval [4] important research topics in the field of intelligent information retrieval technology. The intelligent information retrieval comprehensively uses the retrieval, reasoning, learning and knowledge discovery and other related technologies, on the base of the correct analysis and understanding user needs, effectively find a variety of database and knowledge base to get the knowledge and information of [5-7] user's need. The semantic Web can add the contents that the computer can understand to resources on the Web, and give a method that computer can understand and can represent resources, which is convenient for computer processing. The semantic web architecture as shown in Figure 1, constructed of Web through the concept of machine readable explicit, way, semantic web search architecture is shown in Figure two, so that people can use software agent automatically now needs a lot of human involvement in the work.

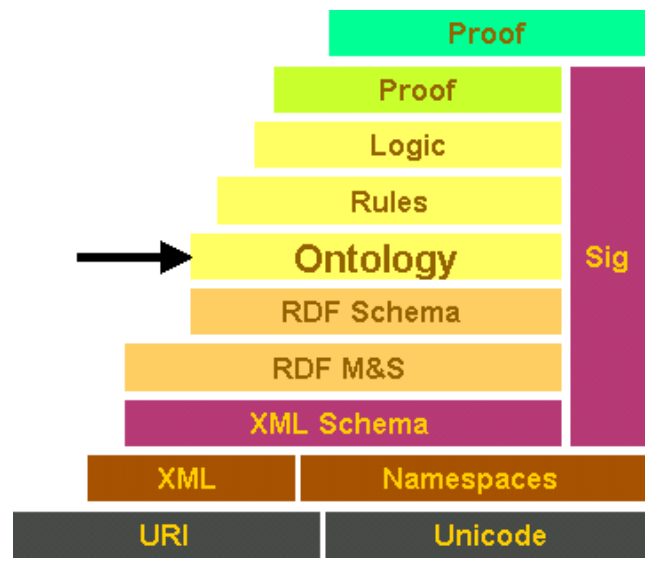

Figure 1: The stack type semantic Web architecture by Berners-Lee

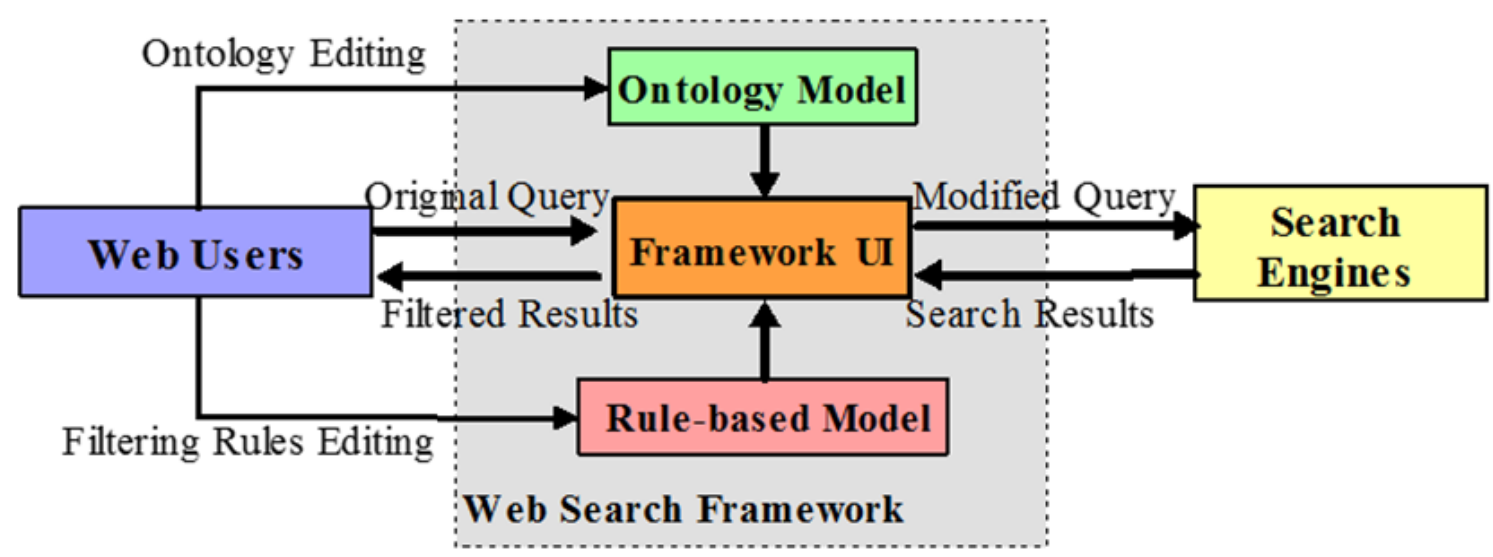

Figure 2: Semantic search framework 
The rules of temporal description logic algorithm based on Ontology are as follows:

$$
1:{ }^{\top}{ }^{I}=\Delta^{I}
$$

2: $\perp^{I}=\Phi$

3: $(\neg C)^{I}=\Delta^{I} \backslash C^{I}$;

4: $(C \sqcap D)^{I}=C^{I} \cap D^{I}$;

5: $(C \sqcup D)^{I}=C^{I} \cup D^{I}$;

6: $(\forall R . D)^{I}=\left\{a \in \Delta^{I} \mid \forall b .\left(b \in \Delta^{I} \wedge R^{I}(a, b)\right) \rightarrow b \in D^{I}\right\}$;

7: $(\exists R . D)^{I}=\left\{a \in \Delta^{I} \mid \exists b .\left(b \in \Delta^{I} \wedge R^{I}(a, b) \wedge b \in D^{I}\right)\right\} ;$

$8(\geq n R . D)^{I}=\left\{a \mid \#\left\{b \mid(a, b) \in R^{I} \wedge b \in D^{I}\right\} \geq n\right\}$ The function \#(set) means to calculate the number for the elements in the collection;

9: $(\leq n R . D)^{I}=\left\{a \mid \#\left\{b \mid(a, b) \in R^{I} \wedge b \in D^{I}\right\} \leq n\right\}$;

10: $\left(R^{+}\right)^{I}=\bigcup_{i \geq 1}\left(R^{I}\right)^{i}$, That means $\left(R^{+}\right)^{I}$ is the transitive closure of $\left(R^{I}\right)$;

11: $\left(S^{-}\right)^{I}=\left\{(b, a) \mid(a, b) \in S^{I}\right\}$;

12: $\left(\exists u_{1}, u_{2} .\left(r_{1} \vee r_{2} \ldots \vee r_{k}\right)\right)^{I}=$

$\left\{a \in \Delta^{I} \mid \exists x_{1}, x_{2} .\left(x_{1} \in \Delta^{T} \wedge x_{2} \in \Delta^{T} \wedge\left(a, x_{1}\right) \in u_{1}^{I} \wedge\left(a, x_{2}\right) \in u_{2}{ }^{I} \wedge\left(\left(x_{1}\right.\right.\right.\right.$,

$\left.\left.\left.x_{2}\right) \in r_{1}^{I} \vee\left(x_{1}, x_{2}\right) \in r_{2}^{I} \vee, \ldots, \vee\left(x_{1}, x_{2}\right) \in r_{k}^{I}\right)\right\}$;

$13\left(\forall u_{1}, u_{2} \cdot\left(r_{1} \vee r_{2} \cdots \vee r_{k}\right)\right)^{I}=$

$\left\{a \in \Delta^{I} \mid \exists x_{1}, x_{2} .\left(x_{1} \in \Delta^{T} \wedge x_{2} \in \Delta^{T} \wedge\left(a, x_{1}\right) \in u_{1}{ }^{I} \wedge\left(a, x_{2}\right) \in u_{2}{ }^{I} \rightarrow\left(\left(x_{1}\right.\right.\right.\right.$,

$\left.\left.\left.x_{2}\right) \in r_{1}^{I} \vee\left(x_{1}, x_{2}\right) \in r_{2}^{I} \vee, \ldots, \vee\left(x_{1}, x_{2}\right) \in r_{k}{ }^{I}\right)\right\}$;

Description: $r_{1}, r_{2}, \ldots, r_{k}$ are atomic predicates in the specific field. If we note $r=r_{1} \vee r_{2} \ldots \vee r_{k}$, then $\exists u_{1}, u_{2} .\left(r_{1} \vee r_{2} \vee \ldots \vee r_{n}\right) \forall u_{1}, u_{2} .\left(r_{1} \vee r_{2} \vee \ldots \vee r_{n}\right)$ can be expressed as $\exists u_{1}, u_{2} . r$ and $\forall u_{1}, u_{2} . r$.

\section{Satisfiability judgment of temporal specific domain}

Algorithm in description logic is used to determine whether a concept is satisfiable, starting from $S_{0}=\{\operatorname{Tr}, G\} . \operatorname{Tr}=\{\{a\}, \phi, \phi, \phi, L\} L(a)=\{C\} G=\{\phi, \phi\}$ Use the algorithm rule repeatedly to extend the complete system $S$, in which nodes are $S$ elements. For notes, if $L(a)$ does not contain a conflict or no rule can be used, for concept, the application of rules makes it construct a complete tree without conflict, then the concept $C$ is satisfiable.

\section{Summary}

Temporal description logic based on ontology is a formal representation based on object knowledge, which is decidable subset of predict logic, used for describing and reasoning the concept of knowledge, has powerful expression ability and good computational properties, so it is also noticeable in the field of knowledge engineering. But the traditional description logic can only model the static knowledge, but can't express temporal knowledge in many applications, hence it is necessary to extend description logic in temporal aspects. This temporal extension method in this paper is based on the concrete domain, which can be used to handle temporal knowledge in the field conveniently. Through the analysis of shortcoming of description logic in processing temporal information, this paper defines time as the temporal specific areas which extends to the description logic, and puts forward a temporal description logic algorithm based on ontology, which to a certain extent, enhances the ability of expression in temporal logic description, and provides a more reasonable logical foundation for the semantic Web. 


\section{References}

[1] Cao Zhisong, Cao Wenjun. Research on effective Web information retrieval based on semantic. Fudan University (Natural science edition), 2004 (3) p. 422-427.

[2] Yan Yalan. The knowledge processing study based on semantic Web, doctoral thesis, 2005.3.

[3] Zhang Yan. Introduction of description logic. Jiangnan University Journal (Natural science edition) 9(1), 2010.2.

[4] Xu Hengnan. Artificial intelligence and intelligent retrieval 2005, 35(1) p.53-54.

[5] Chen Xiaojin, Wang Bing. Intelligent information retrieval method research. p. 83-86.

[6] Huang Guo, Zhou Zhurong, Zhou Ting. Information retrieval research based on semantic web. Southwest University Journal. 2007(29) p.77-80.

[7] Nick Crofts, Martin Doerr, and Tony Gill, etal. Definition of the CIDOC Conceptual Reference Model[R].ICOM/CIDOC CRM Special Interest Group, Version3.4.9, 30th November 2003. 